\title{
LA PERSONA UMANA DI FRONTE AL DIRITTO
}

\section{Luigi Castagnola}

\author{
da Universidade do Paraná
}

Le sorti della persona umana di fronte al diritto furono soggette, lungo la storia, ad una serie di vicende alterne. Dalla più remota antichità fino ai giorni nostri, s'avvicendarono sistemi giuridici, sociali e politici che ora innalzarono la persona umana fino alle stelle, ora la depressero fino alla polvere. Soluzioni così antagonistiche ed opposte dimostrano in modo evidente che non sempre i giuristi, i sociologi ed i politici ebbero lo stesso concetto intorno alla persona umana.

Lo statismo e l'individualismo possono essere considerati come i due poli opposti atorno ai quali gravitarono, a maggiore o minore distanza, le varie dottrine giuridiche che pretesero 0 assorbire l'individuo nello stato o concedergli una così illimitata libertà da comprometterne l'esistenza. L'individualismo prescinde da ogni contatto che il singolo "ha con gli altri e con la società" (1) e attribuisce "alla persona un'autonomia assoluta e ai diritti individuali un valore incondizionato" (2) ; lo statismo radicale ritiene "lo Stato come un soggetto assoluto di diritti e di doveri, al quale deve essere sacrificato l'individuo particolare l'uomo non rappresenta nulla, soltanto lo Stato è tutto" (3).

Esamineremo, in breve sintesi, i pricipali sistemi giuridici costruiti e impiantati dallo statismo; in seguito faremo un bre-

(1) Giuseppe BIEDERLACK, La Questione Sociale, Roma, 1907, p. 21.

(2) Codice Sociale di Malines, Roma, 1944, p. 34.

(3) Guido GONELLA, Bases de uma Ordem Social, Petrópolis, 1947, p. 14. 
ve cenno sull'individualismo esagerato; in fine vedremo come questi due sistemi sono, teoreticamente, rigettati dalla ragione e, praticamente, combattuti dalla retta coscienza delle nazioni.

Lo statismo assoluto sembra il sistema di governo seguito di preferenza tra i popoli antichi, tanto d'oriente che d'occidente. In oriente l'assolutismo potè facilmente affermarsi per il fatto che "la forma teocratica del governo delle antiche nazioni orientali consetiva la maggior oppressione" (4). Infatti i primi grandi aggregati statali che s'incontrano in oriente - come in Assiria, nella Caldea, in Egitto, nella Cina - si presentano governati da capi che vantano sempre qualche parentela con la Divinità. $\mathrm{E}$ siccome la Divinità è concepita con gli attributi dell'eternità, dell'infallibilità, dell'onnipotenza ed esige la riverenza, i reggitori dei popoli, che della Divinità si dicono in qualche forma partecipi, si credono perenni al potere, infallibili e ostentano una tal sovranità sopra gli altri uomini che li fa credere sciolti da ogni freno (5).

Anche in Grecia e a Roma - le cui civiltà ebbero tanta parte nella formazione delle nazioni del mondo occidentale furono misconosciuti i diritti che alla persona derivano dalla natura umana, e, nonostante qualche apparenza in contrario, il "panstatismo fu sempre il sistema preferito di governo" (6).

La ragione di ciò, anchhe qui, va riposta soprattutto "nell'anima profondamente teocratica della città antica. Essendo divinizzate la società, la patria, la sovranità, si era fatalmente condotti ad ampliarne all'infinito i poteri ed i diritti" (7). E questa, infatti, l'opinione di quasi tutti gli studiosi. Federico Ozanam e Fustel de Coulanges sono due autorità degne di es-

(4) Angelo BRUCCULERI, Lo Stato e l'individuo, Roma, 1944, p. 7.

(5) Carattere profondamente teocratico ha pure il diritto mussulmano, che protegge soltanto chi appartiene alla società islamica. Rispetto agl'infedeli "vige teoricamente il puro e semplice stato di guerra; essi dovrebbero essere tutti costretti ad accettare la religione islamica, oppure essere sterminati con la guerra santa". Dizionario Enciclopedico, UTET, Torino, 1936, vol. VI, voce Islamismo, p. 421.

(6) Angelo BRUCCULERI, Lo Stato e l'individuo, cit., p. 8.

(7) Idem, ibidem, p. 8. 
sere ascoltate a rispetto di ciò. Il primo, parlando del mondo romano, scrive: "Essendo divinizzata Roma, le volontà di lei erano tutte divine, legittime e niuna resistenza trovavano le sue leggi nella volontà degli uomini : niuno poteva aver ragione di fronte agli Dei... L'impero è un'idolatria di cui l'imperatore è il sacerdote e il nume a un tempo stesso: gli si innalzano altari pur vivo; ei manda per tutto le sue immagini, e la gente accorre innanzi a quelle con le fiaccole e cogli incensi" (8). Il secondo, parlando della Grecia, potè formulare questo giudizio: “E un errore assai singolare, tra i tanti umani errori, quello d'aver creduto che l'uomo deil'antichità gioisse della libertà. Non se ne aveva neppure l'idea. L'uomo non credeva che potesse esistere un diritto di ïronte alla città e ai suoi Dei... Mutò più volte la forma di governo; ma la natura dello Stato rimase a un ciipresso la stessa, e la sua onnipotenza non diminuì per nulla. Il governo si chiamava ora monarchia, ora democrazia, ora aristocrazia; ma nessuna di queste rivoluzioni diede mai la vera libertà, la libertà individuale. Avere dei diritti politici, votare, designare dei magistrati, potere essere arconte, ecco ciò che chiamavasi libertà; ma l'uomo non era con ciò meno schiavo dello Stato" (9).

Il giurista tedesco, Jellinek, che contestò questa opinione, l'ammise poi implicitamente. Cadde, infatti, in un'implicazione quando relevò che le esagerate esigenze dello Stato sopra l'individuo "erano la conseguenza naturale di una concezione dello Stato, che nelle sue radici rimontava all'antica credenza popolare, la quale vedeva nelio Stato un'opera degli Dei e lo considerava come una permanente patria degli Dei, la cui venerazione costituiva il primo e più elevato dovere del cittadino" (10).

Nel mondo ellenico si concepiva l'individuo, il cittadino, come innestato organicamente nella società e, necessariamente,

(8) Federico OZANAM, La Civiltà nel quinto secolo, Torino, 1871, p. 203.

(9) Fustel DE COUlanges, La Cité antique, Paris, 1905, p. 269.

(10) G. JELLINEK, La dottrina generale dello Stato, Napoli,1921, vol. I, p. 556. 
riceveva "dallo Stato tutte le sue prerogative e dignità. L'uomo non aveva diritti personali e individuali anteriori allo Stato, ma aveva soltando quei diritti che lo Stato gli accordava" (11).

E per essere convinti che in Grecia le cose stessero varamente così, basterà esaminare alcune idee giuridiche e politiche dei due maggiori filosofi di quel popolo così riccamente dotato: Platone e Aristotele.

Platone si occupò dei problemi dello Stato specialmente nei tre dialoghi: La Repubblica, Il Politico, Le Leggi. Nonostante i grandi meriti e l'indiscusso valore di quel nobile pensatore, la sua concezione dello Stato è quella di "uno statolatra" (12). Nella sua Repubblica ideale Platone nega il diritto all'educazione e alla cultura ad una classe di cittadini, i lavoratori, la cui funzione è intieramente materiale: conservare, col lavoro corporale, la vita delle classi superiori. Lo Stato, quindi, non si deve interessare in nessun modo del volgo, della plebe; la schiavitù è un'istituzione necessaria per la società. Platone arriva fino al punto di escludere "dalla natura umana gli uomini inferiori, dediti ai lavori servili" e di considerare "inferiori i barbari, i popoli diversi dal proprio" (13).

La società non è costituita di esseri simili e uguali, ma dissimili e disuguali. L'ordinamento "sociale e politico e il meccanicismo legislativo della Politeia reprimono l'individualismo fino a sacrificare i diritti della persona e le esigenze all'educazione alla personalità" (14). Il filosofo artista pensava che l'individuo doveva scomparire davanti allo Stato e in esso doveva essere assorbito. Giunse perfino ad asserire audacemente, nella Repubblica, che "è lo Stato che dev'essere felice, non l'individuo" (15). Nega ai cittadini il diritto di proprietà ; per impedi-

(11) G. MESSINA, in Civiltà Cattolica, 16 luglio 1955, p. 172.

(12) Umberto PADOVANI, Storia della Filosofia, vol. I, Milano, 1950, p. 175.

(13) Umberto PADOVANI, op. cit., p. 175.

(14) Nicola PETRUZZELLIS, Lineamenti di Filosofia Politica, Bari, 1951, p. 54.

(15) PLATONE, Repubblica, IV, 420. 
re che l'amore per la famiglia e per la prole pregiudichi quello della patria, abolisce il coniugio e la famiglia, ammettendo il comunismo economico e sessuale; per ovviare al pericolo di un soverchio numero di cittadini, il filosofo idealista arriva all'estremo di stabilire con precisione matematica la popolazione della sua utopistica Repubblica. Nelle Leggi stabilisce che il numero dei cittadini della Repubblica deve essere di 5040. Perciò lo Stato dovrà provvedere ad aumentare o a diminuire la popolazione, affinchè sia sempre conservato il numero di 5040 cittadini (16). Indica i mezzi a cui deve ricorrere lo Stato per conseguire questo intento: limitare l'uso del matrimonio, fissare l'età del coniugio dai 20 ai 40 anni per le donne e dai 30 ai 45 per gli uomini (17); i figli illegittimi, i deformi e quelli dei cittadini perversi dovranno essere esposti (18).

Ciò è quanto basta per dimostrare che Platone, uno degli spiriti più illuminati e ben intenzionati dell'antica Ellade, aveva una concezione giuridica, sociale e politica in cui i diritti fondamentali della persona umana erano gravemente miscono sciuti e audacemente negati.

Aristotele tratta della società nella Politica. Lo Stato, anche merose e utopistiche fantasie del suo maestro, na anche nella sua dottrina intorno allo Stato s'incontrano principi che sono gravemente lesivi dei diritti della persona.

Aristotele tratta della società nella politica. Lo Stato, anche per lui, è superiore all'individuo. Ammette come necessaria la schiavitù, e sebbene non neghi allo schiavo la natura umana, gli nega però i diritti politici; e, cosa tanto più grave, nega questi diritti anche al lavoratore, che egli assimila allo schiavo per quello che concerne i diritti politici, non peritandosi di scrivere parole come queste: "La natura ha prodotto i corpi degli uomini liberi diversi da quelli degli schiavi, dando a

(16) PLATONE, Leggi, V.

(17) PLATONE, Repubblica, V, 8.

(18) PLATONE, Repubblica, V. 
questi il vigore necessario per i lavori pesanti della società; facendo invece quelli incapaci a curvare la loro eretta figura a quelle rudi fatiche" (19).

È veramente sorprendente che un filosofo della statura di Aristotele non abbia visto l'ingiustizia della schiavitù. Con rincrescimento il Bonfante è costretto a riconoscere questa grave lacuna nel pensiero aristototelico. Aristotele, scrive l'eminente romanista, usò "di un vero sofisma per difendere l'istituzione condannata, dividendo gli uomini in atti a comandare e destinati a ubbidire: carattere non raro in quell'uomo meraviglioso, ma forse scarsamente dotado di spirito progressista, il che dimostrò pure nel consiglio dato ad Alessandro di governare i greci da re, i barbari da padrone" (20).

Nella Politica, inoltre, Aristotele ammette chiaramente che l'aborto e l'infanticidio sono doveri dello Stato (21).

Questa mentalità giuridica, secondo la quale la legge dello Stato, qualunque essa sia, deve esercitare una forza ferrea su tutti i cittadini, era generale, possiamo dire, presso i Greci. Tanto che è un caso raro, nella cultura greca, incontrare una ripulsa di siffatta concezione giuridica e politica, e restano quasi isolate e solitarie le parole di protesta contro lo statismo legale, di Creonte, messe in bocca da Sofocle alla sua eroina Antigone: "Io non credetti che i tuoi editti avessero tanto valore da far sì che un mortale violi le leggi degli Dei, non scritte ma inviolabile. Chè non ieri nè l'altro ieri furon esse stabilite, ma vigono eternamente e niuno conosce da quanto tempo apparvero" (22).

Non si vuol qui, in nessun modo, negare le veraci conquiste del pensiero greco antico anche nel campo giuridico (23) e

(19) ARISTOTELE, Politica, I, 5.

(20) Pietro BONFANTE, Istituzioni di Diritto Romano, Roma, 1934, p. 41.

(21) ARISTOTELE, Politica, VII, 16.

(22) SOFOCLE, Antigone, vv. 450-460.

(23) È noto che la Grecia, madre della filosofia occidentale, non seppe mai "maneggiare il diritto, e si meritò il rimprovero che le avrebbe poi mosso Vico: certa autem iuris ars Graecis nulla". Cf. G. GRANERIS, Contributi tomistici alla filosofia del diritto, Torino, 1949, p. 2. 
politico; ma la concezione che i Greci ebbero dello Stato, secondo la quale l'uomo era assorbito completamente nell'organismo statale, ripugna al pansiero giuridico e politico moderno, poichè un tale Stato "appare al nostro modo di pensare come un'istituzione tirannica ed esosa" (24).

I Romani non furono filosofi come i Greci, ma si distinsero, tra le altre cose, per quella grande costruzione pratica che è il Diritto Romano. Però non ogni uomo era soggetto di diritti nella società romana; soltanto chi era libero cittadino romano poteva appellare al diritto. Vale a dire, per essere soggetto di diritti si richiedevano come condizioni essenzali: essere libero (avere lo status libertatis) e, quanto ai rapporti di diritto civile, essere cittadino (avere lo status civitatis). Aveva quindi personalità giuridica chi soddisfaceva a queste condizioni.

Non poteva, perciò, essere difeso dal diritto romano chi era schiavo o non era cittadino romano. Il concetto di libertà che vigeva nel diritto romano era ben lontano da quello che ne abbiamo noi moderni, nè corrispondeva al concetto di libertà qual è concepito dalla filosofia razionale. $\mathrm{E}$ così gli schiavi, le donne ed i figli ne erano privi. La libertà giuridica, ossia la "libera facoltà di disporre come si vuole della propria persona e dei propri atti nei limiti stabiliti dal diritto non era nella società antica riconosciuta a tutti gli uomini. Coloro che l'hanno si dicono liberi; coloro che ne son privi affato servi, che noi deiamo schiavi. Lo schiavo non ha nè connubium nè commercium; non è subbietto, ma obbietto di diritti" (25). In virtù del diritto privato romano, lo schiavo poteva essere:

1) venduto dal suo padrone;

2) comprato da chi lo voleva comprare;

3) ucciso da chi aveva potestà su di lui;

4) maltrattato a volontà del padrone;

5) non aveva diritto al matrimonio;

6) non godeva di libertà personale, nè politica;

(24) G. MESSINA, in Civiltà Cattolica, cit., p. 175.

(25) Pietro BONFANTE, Istituzioni di Diritto Romano, cit., p. 40. 
7) non era considerato come cittadino romano;

8) e, in genere, soggiaceva alla sorte delle cose materiali per quello che concerneva i diritti reali.

Non poteva sfuggire al senso di giustizia dei Romani la profonda iniquità di una tale situazione; e, in vero, si escogitarono vari espedienti per rimediare o diminuire gli effetti nefasti che ne derivavano; ma è un fatto che la schiavitù perdurò durante tutto il tempo che Roma fu un governo. Nel diritto romano un uomo poteva essere, in senso stretto, padrone di un altro uomo e farne quello che credeva meglio. Ben è vero che, con l'andar del tempo, specialmente sotto l'influenza dei principi umanitari cristiani, fu mitigata la durezza di leggi così inumane ed inique, ma ciò fu fatto avendo in mira specialmente il bene e l'utilità dello Stato. Così è che, nell'età imperiale, il legislatore s'interessò della condizione dello schiavo, per mitigarla, guidato in ciò da un sentimento etico, ma soprattutto da interesse politico. Infatti l'espansione "conquistatrice di Roma aveva influito in guisa terribile ad aumentare il numero degli schiavi e abbassarne la condizione; onde le ribellioni, che una volta misero quasi a repentaglio l'esistenza stessa dello Stato. Augusto tentò d'infrenare gli abusi dei padroni con provvedimenti di polizia, affidando al praefectus urbi la vigilanza su schiavo e padroni: una Lex Petronia del 19 d. C. vietò di esporre gli schiavi alle fiere, salvo per decisione del magistrato, Claudio sancì la perdita del diritto sullo schiavo, se infermo era abbandonato dal padrone" (26).

Da ciò si può vedere fino a che punto si era giunti nel disprezzo dei diritti della persona umana nella società romana.

Inoltre, lo straniero, detto nelle XII Tavole hostis, nel diritto posteriore peregrinus, non era difeso dal diritto perchè non era cittadino romano.I filiifamilias, nel campo del diritto privato, non godevano "di veruna capacità attuale per l'acquisto di diritti" (27). La capacità giuridica della donna era "per

(26) Pietro BONFANTE, op. cit., pp. 171-172.

(27) Pietro BONFANTE, op. cit., p. 54. 
più riguardi inferiore a quella dell'uomo. La donna è incapace di diritti politici e d'ogni funzione pubblica" (28).

Nel mondo antico, dunque, tanto in quello greco che in quello romano, per non parlare dei cosiddetti popoli barbari, i diritti della persona umana erano crudamente negati.

Con lo sfasciarsi dell'impero romano non tramontò sul cielo della storia il concetto della statolatria. Per quel che riguarda la schiavitù, si deve riconoscere che essa fu praticata anche in paesi cattolici, contro lo spirito della dottrina cristiana. Paesi cristiani come la Spagna e il Portogallo, la introdussero nelle colonie americane per motivi economici, sebbene in una forma assai più mitigata che non fosse quell'antica. Ma, poco per volta, grazie al profondo senso di giustizia dei popoli americani, una volta che essi ebbero conquistata l'indipendenza, abolirono un'istituzione così antiumana. E ciò costituisce uno dei non pochi titoli di gloria di cui si coprirono le grandi repubbliche democratiche delle nobili Americhe, dove tali conquiste umanitarie in difesa dei diritti della persona umana furono raggiunte sotto l'influsso benefico del sentimento di giustizia e carità portato nel mondo dal messaggio cristiano.

L'ambizione assolutista continua ad affiorare, di tanto in tanto, sull'orizzonte della storia, ora instaurata da capi dispotici - siano essi re, imperatori o presidenti - ora insegnata da giuristi e filosofi. Esamineremo il pensiero di tre ardenti fautori del panstatismo, sempre contrario ai diritti della persona umana: un italiano (Nicolò Machiavelli), un inglese (Tommaso Hobbes) e un tedesco (Giorgio Hegel).

L'umanesimo italiano dei secoli XV e XVI, per molti aspetti, volle essere un ritorno alla vita sociale precristiana in tutte le sue forme. Toccò a Nicolò Machiavelli la sorte, poco onorevole, di inaugurare all'epoca della Rinascenza la concezione della nuova politica del panstatismo, formulandone la dottrina, in modo frammentario, con stile nervoso, deciso, scheletrico, audace specialmente nelle famose sue opere: Il Principe (1513) e i Discorsi sopra la prima Deca di Tito Livio (1521).

(28) Pietro BONFANTE, op. cit., p. 57. 
Machiavelli (1469-1527) non fu un giurista e, in verità, neppure un vero filosofo (29). Tuttavia si interessò con passione di cose politiche e ne scrisse con abilità di grande letterato. Qua e là non lascia di far vedere quali fossero i suoi pensieri in materia politica, ed è in base a questi sparsi frammenti ideologici che si può costruire il suo mondo politico, e si può intravedere la struttura delle sue concezioni etiche.

Per Machiavelli una cosa è certa: lo Stato è il valore supremo e tutto deve essere subordinato all'organizzazione, alla conservazione e all'ingrandimento dello Stato. Se i Discorsi sulla prima Deca di Tito Livio “rappresentano un'espressione attenuata del pensiero machiavellico e una mitigazione di alcune sue tesi, il principio statolatrico è tuttavia chiaro e indubbio: morale e religione sono semplicemente strumenti della prosperità e della grandezza dello stato, instrumenta regni" (30). Il Principe, ossia il capo dello stato, è libero da ogni legge etica e giuridica - che però può anche osservare, quando ciò gli sia vantaggioso - nella formazione e conservazione dello Stato. Potrà e dovrà il Principe essere giusto o ingiusto oservare il diritto o violarlo, promettere e non mantenere, invitare a banchetto i suoi avversari e ucciderli a tradimento, sembrare onesto e virtuoso senz'esserlo veramente, purchè ciò giovi allo Stato, rispettare la religione o combatterla secondo che convenga. Tutte queste cose sono tollerate e ammesse da Machiavelli. Faccia "dunque uno principe di vincere e mantenere lo Stato:

(29) A proposito scrisse Nicola Petruzzellis: "Anche per il Machiavelli sussiste la pregiudiziale se possa a rigore di termini considerarsi filosofo e sia pure filosofo della politica, come suona un autorevole luogo comune. Ora, manca al pensiero machiavellico quel contenuto e quel valore universale, che costituiscono e differenziano la filosofia. Anche dagli apologisti del Machiavelli, che non esitano ad annoverarlo tra i filosofi, si suole ammettere che egli prescinde dal problema metafísico e dal problema cosmologico, nonchè, si può aggiungere, da quello gnoseologico, ma si rileva che non prescinde da quello etico. Ma non si tien conto in queste asserzioni che il Machiavelli ne prescinde non già per motivi contingenti, ma per i caratteri intrinseci del suo pensiero, alieno dagli orizzonti e dalla disciplina della speculazione filosofica. Il problema etico-politico non è suscettibile di una soluzione filosofica, se non in un intimo rapporto con gli altri problema della filosofica". Cf. Nicola PETRUZZELLIS, Lineamenti di Filosofia della Politica, cit., pp. 108-109.

(30) Nicola PETRUZZELLIS, op. cit., p. 115. 
e'mezzi sempre saranno iudicati onorevoli e da ciascuno laudati: perchè el vulgo ne va preso con quello che pare, e con lo evento della cosa; e nel mondo non è se non vulgo" (31).

Questo immoralismo politico trovò larga fortuna e numerosi seguaci dopo Machiavelli, tanto nella pratica che nella teoria. Ancora recentemente ci fu chi osò scrivere parole come queste, ispirandosi alla concezione politica del famoso segretario fiorentino: "I precetti "non uccidere" e "non rubare", che sono i pilastri di ogni etica naturale oltre che rivelata, non si possono ammettere in politica. Applicati alla lettera significherebbero la condanna e l'abolizione della guerra, che è la stessa vita dello Stato... Il delitto politico non è, propriamente, un delitto. La morale lo condanna con la sua legge; la politica lo scusa in virtù di un'altra legge, che è quella della salvezza dello Stato" (32).

È poi evidente che in una concezione statolatrica dello Stato, in cui la morale è completamente sottomessa alla politica, non si può più parlare di diritti della persona umana. I mezzi violenti e immorali, che Machiavelli consigliò al suo Principe di praticare inflessibilmente per la formazione e la conservazione dello stato, gettarono "un'ombra sul suo nome e sulla sua opera" (33).

In Inghilterra fautore tipico dello statismo, anzi dell'assolutismo monarchico fu Tommaso Hobbes (1588-1679), le cui dottrine giuridiche e politiche sono espresse specialmente nel $D e$ Cive (1642) e nel Leviathan (1651).

Il filosofo inglese arriva alla sua dottrina dell'assolutismo statale attraverso una singolare concezione della società. Come è noto, la società, secondo Hobbes, risulterebbe da un accordo

(31) Nicolò MACHIAVELLI, Il Principe, XVIII, 5.

(32) G. MAgGIORE, La Politica, Bologna, 1941, pp. 31-32.

(33) G. DEL VECCHIO, Lezioni di Filosofia del Diritto, Milano, 1946, p. 50.

(34) T. HOBBES, De Cive, c. 12, n. 1. 
nato tra i cittadini, i quali, per uscire da uno stato selvaggio presociale, metterebbero tutti i diritti e tutti i poteri dei singoli nella mani del sovrano affinchè questi provveda alla conservazione della pace sociale. Prima della costituzione di una tale società e della stipulazione di un tale accordo non esistevano nè diritti nè doveri, nè v'erano cose ingiuste o giuste (34). Dopo l'accordo tra i cittadini ed il sovrano, il popolo ed i singoli sono abbandonati all'arbitrio del monarca (35).

Il diritto, per conseguenza, è determinato della voluntà dispotica del sovrano. Egli determina ciò che è giusto e ciò che è ingiusto. Hobbes dice con estrema audacia che i sovrani "quae imperant iusta faciunt, quae vetant iniusta" (36). Qualunque cosa il principe comandi sarà necessariamente giusta per il semplice motivo che è comandata da lui. Parole come queste fanno oggi l'effetto di una sassata.

Naturalmente, in una simile concezione sopra l'origine della soicetà e del diritto, non c'è più posto per $\mathrm{i}$ diritti della persona umana.

L'errore fondamentale di Hobbes, scrive Giorgio Del Vecchio con l'autorità che gli è propria, è "alla radice, e consiste nella limitazione arbitraria della natura umana all'egoismo" (37). Ma si deve osservare che anche la concezione della società, ammessa da Hobbes, è assolutamente fantastica e contradetta dalla ragione.

Doveva toccare, però, alla Germania il primato nella elaborazione della più assoluta dottrina statolatrica, per opera del filosofo idealista Giorgio Hegel (1770-1831). Il pensiero giuri..........

dico e politico di Hegel è esposto specialmente nelle sue due opere: Fenomenologia dello spirito (1807) e Filosofia del diritto (1821).

(35) T. HOBBES, De Cive, c. 6, n. 16.

(36) T. HOBBES, De Cive, c. 12 , n. 1.

(37) G. DEL VECCHIO, Lezioni di Filosofia del Diritto, cit. p. 59. 
Tutti sanno che la dottrina fondamentale che Hegel pose a base della sua concezione del diritto, dello stato e della società è l'idealismo assoluto o panlogismo. Non essendo possibile, per motivi pratici, esporre qui tal dottrina, è essa supposta. Avvertiamo, però, che non si può comprendere il pensiero giuridico e politico di Hegel senza la conoscenza del suo panlogismo.

Che pensa Hegel dello Stato? Secondo il filosofo tedesco "lo Stato è addirittura Dio stesso, e come tale non ha nè può avere alcun limite al suo potere. Lo Stato è la suprema realtà in cui s'individua lo spirito del mondo e l'etica stessa, che non riconosce ma crea il diritto" (38). Se, in apparenza, la concezione giuridica di Hegel consacra la dignità umana, in realtà sottomette l'individuo allo Stato senza possibilità di scampo. L'assolutismo hegeliano è perfetto e non ha nulla da invidiare agli Stati più tirannici dell'antichità. Non si poteva, invero, concepire "una forma di assolutismo più brutale di quella in cui l'individuo, la famiglia, l'ordine civile, morale e religioso, la scienza e l'arte, tutto, in una parola, fosse subordinato allo Stato. Ora, posto il principio che lo Stato è tutto, che l'individuo è nulla, è vano parlare ancora di un diritto alla libertà" (39).

L'influenza di Hegel è stata grande sulla cultura europea e pertanto, anche sulla vita politica delle nazioni del Vecchio Mondo, dove ambiziosi capi di stato tentarono, più volte, l'applicazione pratica dell'idea statale hegeliana. Ma ogni volta che ciò fu tentato, non si tardó a raccogliere sulle rovine ammucchiate da così nefasta ideologia "fiori di cenere e tosco". $\mathrm{E}$ dopo l'ultima guerra mondiale, indetta da uno spirito che voleva sottomettere l'umanità intera al ferreo arbitrio dello Stato, ma vinta da quelli che lottarono fino all'esasperazione pur di liberarsi da così inumane catene, non mancarono illuminati cultori della filosofia del diritto, che accusarono la dottrina dell'assolutismo statale qual principale causatrice di tante sofferenze alle nazioni della nostra epoca. "Já se fêz, no terreno histórico, a prova desta doutrina... o Estado absoluto, que exige a bôlsa

(38) A. BRUCCULERI, Lo Stato e l'individuo, cit., p. 16.

(39) G. B. BIAVASCHI, La Moderna concezione filosofica dello Stato, Milano, 1923, pp. 175-176. 
e a vida, o dinheiro e o sangue, que - sonegando a homem o direito de opinar - fixa por decretos o que é verdadeiro e o que é falso, e chega ao extremo de determinar o que se deve amar e o que odiar" (40). "Sustentando, com Hobbes, Hegel e seus retardados e monótonos repetidores, que o Estado é o todo, o divino que se encarna no mundo, que é o espírito da história, o moderno laicismo político se fêz pregoeiro de uma teocracia temporal que, divinizando o Estado, atribui absolutismo, infalibilidade e onipotência ao principado. Todos os modernos Estados absolutos, tôdas as formas de barbárie que se adornam com o rótulo de pagãs, divinizam ou o príncipe ou o povo e em seu nome se tornam tiranas do homem, da sua liberdade, dos seus bens e, afinal, da sua consciência, ignominiosamente constrangida à vileza do conformismo" (41).

La storia recente, infatti, è la dimostrazione palmare dell'errore che si annida nella concezione del panstatismo, negatore dei diritti della persona umana. Si spiega, così, come capi ambiziosi e dominati da un egoismo sfrenato ricorrano a tale dottrina per dissimulare, sotto le parvenze della ragione di Stato, la loro sete di potere, che Nietzsche definì "volontà di dominio", ma che, in realtà, altra cosa non è che "a implantação do orgulho, do instinto e, portanto, da desapiedada fôrça física" (42).

In questi ultimi tempi apparve una parola nuova per indicare l'onnipotenza statale ed ebbe larga fortuna: il totalitarismo statale. Nato in Italia, questo neologismo fece il giro del mondo in pochi anni. Sebbene il suo significato sia stato stiracchiato in tutti i sensi e adottato da regimi politici apparente-

(40) Guido GONELLA, Bases de uma Ordem Social, Petrópolis, 1947, pp. 124-125.

(41) Guido GONELLA, Bases de uma Ordem Social, cit., p. 118.

(42) Guido GONELLA, Bases de uma Ordem Social, cit., p. 158. Ancora recentemente, una ripulsa dell'assolutismo statale di Hegel, inacettabile per le conseguenze a cui porta, è stata fatta, in un eruditissimo lavoro originale, da José Nicolau dos Santos. Cf. José Nicolau DOS SANTOS, ONU - Estado, Proto-Estado ou Super-Estado?, Curitiba, 1952, pp. 118-119, nota 1 . 
mente in contrasto, esso indica, nel complesso, "quel sistema teorico o metodo ed indirizzo pratico, che dà allo Stato un potere illimitato, o pressochè tale, sull'individuo, isolato o associato, in guisa da sconoscerne in tutto o in parte i suo naturali diritti" (43). Le tre forme più conosciute del totalitarismo di stato, concretizzate in vari paesi in questi ultimi tempi, sono: il fascismo in Italia, il nazionalsocialismo in Germania, il comunismo in Russia.

Sia che questo totalitarismo sia l'espressione della volontà di un solo, sia che si presenti fintamente sotto l'aspetto della volontà della massa popolare, artificiosamente sobillata, esso viola sempre i fondamentali diritti della persona umana. Sarà sufficiente citare alcuni passi presi dai principali autori delle tre suddette ideologie politiche perchè sia evidente come, in ognuna di esse, sia abolita la libertà individuale e celebrata la oppressione fisica di tutto ciò che non è conforme alla volontà di chi governa.

Brani tolti da Lenin e da Stalin:

"Il proletariato ha hisogno del potere statale, di un'organizzazione centralizzata del potere, sia per schiacciare la resistenza degli sfruttatori, sia per guidare l'immensa massa della popolazione, i contadini, i piccoli borghesi e i semiproprietari, nel lavoro costruttivo dell'ordine economico socialista" (44).

"La dittatura del proletariato implica una serie di restrizioni della libertà degli sfruttatori, degli oppressori, dei capitalisti. Noi dobbiamo opprimerli per liberare l'umanità dalla schiavitù del salario. La loro resistenza deve essere spezzata con la forza, ed è evidente che dove ha luogo una repressione, dove è adoperata la violenza, non esiste democrazia. La democrazia per la stragrande maggioranza del popolo è la repressione violenta degli sfruttatori, degli oppressori del popolo, cioè la loro esclusione dalla democrazia" (45).

(43) Angelo BRUCCULERI, Lo Stato e l'individuo, cit., p. 7.

(44) LENIN, Stato e Rivoluzione, Milano, 1920, p. 29.

(45) LENIN, Stato e Rivoluzione, cit., p. 99. 
"La dittatura, nella sua concezione scientifica, non significa altro che un potere non limitato da nessuna legge; non ostacolato affatto da alcuna regola e si fonda direttamente sulla violenza... La dittatura importa (notatelo bene una volta per sempre, miei signori cadetti), il potere illimitato che si regge sulla forza e non sulla legge" (46).

"La dittatura del proletariato è la guerra più eroica e più implacabile della classe nuova contro un nemico più potente, contro la borghesia... la dittatura del proletariato è la lotta tenace, sanguinosa e non sanguinosa, violenta e pacifica, militare ed economica, pedagogica ed amministrativa contro le forze e le tradizioni della vechia società" (47).

Passi tolti dagli scritti di Mussolini:

"Per il fascista, tutto è nello Stato, e nulla di umano o spirituale esiste, e tanto meno ha valore, fuori dello Stato. In tal senso il fascismo è totalitario, e lo Stato fascista, sintesi e unità di ogni valore, interpreta, sviluppa e potenzia tutta la vita del popolo" (48).

"Nè individui fuori dello Stato, nè gruppi (partiti politici, associazioni, sindacati, classi)" (49).

"Lo Stato, infatti, come volontà etica universale, è creatore del diritto" (50).

"Per il fascismo lo Stato è un assoluto, davanti al quale individui e gruppi sono il relativo. Individui e gruppi sono pensabili in quanto siano nello Stato" (51).

"Ma v'è un lato della educazione nel quale noi siamo, e non si vuol dire intrattabili, intransigenti... Dire che l'istruzione spetta alla famiglia, è dire cosa al di fuori della realtà contem-

(46) LENIN, De l'Etat."Contribution à la question de la dictature". Citato da STALIN: Question de leninisme, Paris, 1936, p. 22.

(47) STALIN, Principii del leninismo, Roma, 1943. pp. 29-31.

(48) MUSSOLINI, La Dottrina del fascismo, parte I, paragrafo VII.

(49) Idem, ibidem, paragrafo VIII.

(50) Idem, ibidem, paragrafo $\mathrm{X}$.

(51) Idem, ibidem, parte II, paragrafo $X$. 
poranea... Solo lo Stato, con i suoi mezzi di ogni specie, può assolvere questo compito. Aggiungo che solo lo Stato può anche impartire la necessaria istruzione religiosa" (52).

“... nello Stato la Chiesa non è sovrana e non è nemmeno libera. Non è sovrana per la contradizion che nol consente: non è nemmeno libera, perchè nelle sue istituzioni e nei suoi uomini è sottoposta alle leggi generali dello Stato" (53).

Il nazionalsocialismo hitleriano, conosciuto anche col nome di "razzismo", pretese inaugurare una nuova concezione dello stato: esso sarebbe soltanto un mezzo per raggiungere e conservare la purezza del sangue. La conservazione della razza nazionale è l'unica suprema finalità dello stato nazionalsocialista (54).

(52) Scritti e discorsi di B. Mussolini, vol. VII, pp. pp. 111-112, Milano, 1935.

(53) Scritti e discorsi di B. Mussolini, vol. VII, p. 32, Milano, 1935.

(54) "Recens est error germanicus hitlerismi secundum quem functio et finis Status habentur unice et vere exclusive in tuenda et servanda puritate sanguinis seu gentis (razza); omnia alia problemata socialia sunt nugae si comparantur cum hoc quod est unicum, verum et praecipuum problema: scil. quaestio de conservanda indole et puritate stirpis nationalis". Alaphridus OTTAVIANI, Compendium Iuris Publici Ecclesiastici, Romae, 1936, p. 288. Cf. HITLER, Mein Kampf, München, 1933 , pp. 420; 359; 360. Circa la dottrina nazionalsocialista, cfr. HITLER, Mein Kampf, München, 1933, specialmente le pagine 451 e ss., 490, 501; ROSENBERG, Der Mythus des XX. Jahrhunderts, Müchen, 1933, pp. 117-118. Le dottrine dell'hitlerismo circa lo Stato e l'individuo furono sintetizzate come segue dalla Civiltà Cattolica, Roma, 1934, vol. II, p. 358: "Sull'individuo lo Stato ha tutti i diritti: di mutilarlo e di sopprimerlo, di sbandirlo e di opprimirlo, di chiamarlo alla partecipazione della vita pubblica o di negargli i diritti civili. La fonte unica di tutti i diritti è la razza, e quindi lo Stato, il quale concede all'uomo certe facoltà, che può anche ritirare quando vuole e come vuole, senza rispetto alcuno alla persona umana".

E un fatto degno di essere rivelato, che in Roma, nell'Institutum Utriusque Juris, si combattevano dalla cattedra gli errori filosofici e giuridici delle varie forme di totalitarismo quando esso era nel suo auge e lo si combatteva con alto spirito giuridico. I fatti storici, pochi anni dopo, dovevano incaricarsi di dimostrare quanto l'indirizzo dell'insegnamento giuridico di quella Facoltà romana di scienze giuridiche fosse giusto. Oggi tutte le nazioni democratiche del mondo sconfessano apertamente i sistemi totalitari, perchè gravemente contrari al benessere dei cittadini. 
Ecco alcuni passi tolti dal libro Mein Kampf di A. Hitler :

"La concezione nazionale razzista riconosce il valore dell'umanità nei suoi primordiali elementi di razza. In conformità dei suoi principi, essa ravvisa nello Stato soltanto un mezzo per raggiungere un fine, il fine della conservazione razzista degli uomini" (55).

"Tutte le grandi questioni che si presentano in un dato tempo sono soltanto questioni del momento e sono soltanto conseguenze di certe cause. Fra loro una sola è veramente di importanza principale: la questione della conservazione della razza nazionale. Nel sangue solo è fondata la forza e anche la debolezza di un popolo" (56).

"Lo Stato deve fare in modo che chi è sano generi figli; che sia scandaloso mettere al mondo bambini quando si è malati o difettosi ; e che nel rinunziare a ciò consista il supremo onore. $\mathrm{Ma}$, viceversa, dev'essere ritenuto riprovevole il sottrare alla nazione bambini sani. Quindi lo Stato deve presentarsi come il preservatore di un millenario avvenire, di fronte al quale il desiderio e l'egoismo dei singoli non contano nulla e debbono piegarsi" (57).

"Lo Stato nazionale deve, in questo riconoscimento del suo complessivo lavoro di educazione, mirare in prima linea non ad infondere una semplice volontà ma ad educare corpi sani. Solo dopo, in seconda linea, viene lo sviluppo della capacità spirituale...

Lo Stato può intraprendere anche l'educazione delle ragazze, partendo dagli stessi pundi di vista in cui si mette per svolgere l'educazione dei ragazzi. Anche qui si deve attribuire la maggiore importanza all'educazione del corpo, e solo dopo si deve pensare a promuovere i valori psichici e intellettuali"(58).

(55) Cfr. HITLER, La mia battaglia, tr. it., Milano, 1934.

(56) HITLER, Mein Kampf, cit., p. 372.

(57) HITLER, op. cit., II, pp. 446-448.

(58) HITLER, op. cit., pp. 451-459. 
È poi evidente che, davanti a tali concezioni sulla natura e finalità della società, dello stato, della famiglia, dell'individuo non si può più parlare di libertà e di diritti della persona umana. Queste dottrine totalitarie hanno l'errore comune di erigere a valore supremo cose che hanno bensì un valore ma soltanto relativo. Ed hanno ancora l'errore di metodo, nel volere impiegare la forza fisica, la violenza per imporre nella pratica sistemi di vita sociale irrazionali e, per conseguenza, antiumani (59).

Si oppone allo statismo l'individualismo, che suole apparire come una rivalsa dello spirito di libertà, insito naturalmente nell'uomo, contro le ingiuste imposizioni legaliste.

L'individualismo di cui s'intende qui parlare è quello che vuole emancipare l'uomo da qualunque vincolo sociale, come pure da ogni legge morale ed etica. Emancipazione completa, quindi, tanto nel campo economico, che in quello etico e politico. In questo sfrenato individualismo trova legittima cittadinanza anche l'anarchia, che ne è la forma estrema. L'anarchico, infatti, è colui che vive "fora da lei, aquêle que faz o que lhe agrada... nega a lei porque nega todo limite à expansão da sua individualidade, que, na sua opinião, deverá afirmar-se livre de qualquer disciplina interior e exterior. Esta suposta liberdade (que melhor se chamaria licença) é uma característica das formas extremas do individualismo" (60). Siffatto spirito individualista arriva a condannare rigidamente l'organizzazione po-

(59) Non si vuol sostenere qui che tutti gli autori di ideologie assolutiste siano nemici dichiarati della loro patria o dell'umanità, in generale. Unicamente si vuole mettere in evidenza, tanto nel campo teorico che in quello pratico, tanto nel campo giuridico come in quello politicu, l'errore della dottrina, prescindendo dalle intenzioni particolari dei loro autori. La storia, che è davvero, come disse già anticamente Cicerone, magistra vitae, dimostra che, molte volte, fu l'incomposta e libertaria condotta dei popoli a generare la tirannide. Se per libertà s'intende il diritto di mettere sullo stesso piede di uguaglianza la giustizia e l'ingiustizia, la verità e la menzogna, è chiaro che si prepara, ineluttabilmente, la tirannia. Cfr. A. BRUCCULERI, La Democrazia, Roma, 1946, pp. 44-52. Come scrisse un eminente studioso: "La democrazia si basa sulla speranza di certe virtù nel popolo e sulla sua tendenza a nutrirle e svilupparle". Cfr. James BRYCE, Democrazie moderne, Commento critico e conclusioni generali, Milano, 1931, p. 473.

(60) Guido GONELLA, Bases de uma Ordem Social, cit., p. 209. 
litica, considerata sempre soffocatrice delle energie dell'individuo. Gli anarchici o libertari rigettano lo Stato soprattutto perchè "non si può ammettere alcun potere coercitivo sull'uomo. Se è proprio dell'essenza dell'uomo l'esercizio della ragione che si crea liberamente la sua legge, non può affatto giustificarsi un'autorità coercitiva dell'uomo al di fuori dell'uomo" (61). $\mathrm{E}$ di fatto scrisse il paranoico esaltatore del superuomo: "Stato si chiama il più freddo di tutti i mostri. È freddo pur nel mentire; e questa è la menzogna che esce dalla sua bocca: Io, Stato, sono il popolo... Lo Stato è là dove tutti, buoni e cattivi, si ubbriacano di veleno: dove tutti si smarriscono: dove il lento suicidio di tutti si chiama la vita" (62).

Se questa forma di individualismo totalitario (l'individuo è tutto) può avere una spiegazione come reazione contro la prepotenza dello Stato totalitario (lo Stato è tutto) (63), non è però giustificabile teoricamente, poichè concepisce l'individuo in modo astratto, sprovvisto della sua nota fondamentale della sociabilità naturale. Non si può negare la legittimità e necessità dello Stato in nome della libertà dell'individuo, nè la vera libertà dell'individuo (e delle giuste associazioni particolari) in nome dello Stato dispotico. Gl'individualisti assoluti cadono nell'errore di considerare l'uomo come un essere completamente autonomo da ogni legge, come "uma nômada sem portas nem janelas, aspirando a uma liberdade absoluta, que o Estado, seu inimigo, procura circunscrever por um solerte e intolerante sistema de limitações coercitivas. 0 indivíduo é um bem, e o Estado um mal. Portanto: guerra ao Estado, para a negação total do Estado" (64).

La libertà politica, svincolata da ogni etica, diventa il veicolo di passioni incomposte, sottratte al controllo della ragione, cieche ed egocentriche. In tal modo si arriva a perturbare, nel-

(61) Angelo BRUCCULERI, Lo Stato e l'individuo, cit., p. 24.

(62) Federico NIETZSCHE, Così parlò Zarathustra, Milano, 1925, pp. 85-86.

(63) Fu, però, anche rilevato che gli accessi demagogici dell'individualismo fecero esplodere violente reazioni in molti paesi europei. Cfr. A. BRUCCULERI, La democrazia, cit., p. 103.

(64) Guido GONELLA, Bases de uma Ordem Social, cit.; p. 175. 
la lotta politica, l'ordine sociale, finchè "la sfrenata libertà politica degli uni si converte nella inevitabile sopraffazione degli altri e diventa tirannide" (65).

Tale individualismo, nel suo vano sforzo di emancipare l'individuo da ogni vincolo sociale, finisce per negare la dignità della persona umana e termina sacrificando il tutto (la società) alla parte (l'individuo). E così, invece di liberare l'uomo, questo falso individualismo, spogliando la persona umana dei suoi valore fondamentali, ne diventa il suo oppressore. Strana, ma comprensibile "ironia de uma doutrina que, apregoada como defensora do homem, acabou por gerar o mito do superhomem, ou seja dêsse ser monstruoso (do qual os fatos contemporâneos nos oferecem exemplares vivos, estúpidos e cruéis) que se sobrepõe à comunidade dos homens e a esmaga em nome de repudiáveis e inumanos mitos (raça, supremacia, etc.) (66).

Da ciò che precede si può concludere che, tanto lo statismo quanto l'individualismo assoluto, sono sistemi giuridici e politici i quali, nel loro antagonismo radicale, negano o pregiudicano i diritti fondamentali della persona umana, le cui caratteristiche essenziali sono la sostanzialità, l'individualità e la razionalità.

Se l'uomo esiste prima dello stato, ha certamente dei diritti che non ha ricevuto dallo Stato e che, per conseguenza, questi non gli può togliere con la violenza. Lo stesso si dica della famiglia. Anch'essa precede lo Stato, nell'ordine cronologico dell'esistenza delle cose. Per altro, l'individuo è un essere sociale per natura, e perciò, col pretesto di una malintesa libertà, non può l'individualismo esagerato negare l'esistenza della società e dell'autorità. $E$ siccome non è possibile raggiungere la finalità desiderata e necessaria - il bene comune - senza un'autorità che coordini le volontà molteplici, è pur necessario lo Stato.

(65) Nicola PETRUZZELlis, Lineamenti di Filosofia Politica, cit., p 194:

(66) Guido GONELLA, Bases de uma Ordem Social, cit., p. 13. 
L'uomo, dotato di ragione com'è, deve saper conoscere i diritti essenziali dei vari elementi che formano la società - individuo, famiglia, stato; ma deve pure conoscere i limiti di tutti questi diritti. È compito di una sana e chiaroveggente filosofia del diritto e della politica conoscere, teoricamente, i diritti dell'individuo e dello Stato, come è compito della sapienza legislativa compilare codici - e via via modificarli, in virtù delle nuove esigenze imposte dal dinamismo umano - in cui quei diritti siano rispettati nella pratica.

Quando c'è non lotta sopraffatrice, ma armonia e rispetto, tra i diritti dell'individuo e dello Stato, un paese vive in pace e il popolo, con serenità ed efficienza, costruisce l'edificio del suo benessere individuale e collettivo, e la sua storia si svolge con ritmo progressista.

La storia antica e quella recente dimostra che i regimi totalitari e quelli individualisti ad oltranza furono, dopo più 0 meno lunga esperienza, combattuti, rigettati e sostituiti da for. me democratiche di governo. Per esempio, furono le intemperanze dell'individualismo libertario che portarono, in epoca recente, vari popoli d'Europa a instaurare sistemi di governo autoritari, dittatoriali, che esplosero come reazioni. "In Ispagna si ebbe quella del falangismo nel 1933, in Austria si crea la costituzione cristiana federale corporativa del 1934, in Polonia si ha il colpo di Stato del 1926 e si adotta la costituzione a carattere presidenziale del 1935, in Lettonia s'insorge col colpo di Stato e l'abolizione del parlamento del 1926, in Estonia cessa nel 1934 di applicarsi la costituzione, in Grecia nel $1936 \mathrm{Me}-$ texas scioglie il parlamento e instaura un regime d'autorità. Più radicale fra tutte è la reazione del fascismo in Italia e, peggio ancora, quella del nazismo in Germania" (67). Nè mancarono esempi simili nei paesi latinoamericani.

Per altro, questi regimi a carattere dittatoriale, nati per ovviare ai disordini causati dall'individualismo, scivolarono all'estremo opposto di togliere la libertà ai cittadini, e finirono

(67) Angelo BRUCCULERI, La Democrazia, cit., p. 103. 
per violare in modo tragico e tirannico i diritti della persona umana. Furono, perciò, combattuti e rigettati anch'essi dalla retta coscienza delle nazioni, che si orientò rapidamente verso forme democratiche di governo, in cui la libertà politica permette il pluralismo dei partiti. La democrazia importa, necessariamente, la lotta partidaria, che "se assai spesso non è senza qualche inconveniente, val la pena tollerarla, per non perderne i vantaggi" (68). Questi non potranno mancare se la lotta partidaria sarà disciplinata e razionale, poichè, in tal caso, non sara perniciosa, bensì "condizione d'ogni progresso ed esigenza incoercibile" (69) della natura umana.

Stato e individuo sono necessari, ma debbono entrambi rispettare i diritti reciproci. In sintesi : lo Stato non deve opprimere, ma tutelare i diritti dell'uomo, e l'individuo ha l'obbligo di raggiungere i suoi fini in armonia con quelli della società, rispettando i diritti dello Stato e degli altri cittadini. Il criterio oggettivo che deve limitare tanto la volontà statale che quella indiduale, deve essere la legge morale, alla quale sono entrambi sottomessi. Lo Stato non limita l'individuo, nè l'individuo limita lo Stato, ma la legge etica limita ambedue, poichè determina la sfera del lecito e dell'illecito e impone alla volontà statale e a quella individuale il limite dei propri diritti. Se lo Stato viola i diritti della persona umana, fugge al suo dovere ed autorizza l'individuo ad apporsi allo stato ed a "não mais reconhecer o direito do Estado, a não se sentir mais vinculado aos seus deveres individuais para com o Estado. Para reclamar respeito aos próprios direitos, precisa cumprir seus próprios deveres; e o Estado, quando desconhece o seu dever de servir ao homem, renuncia implìcitamente ao seu direito de mandar, pois o poder do Estado sôbre o indivíduo è justificado sòmente pela assistência

(68) Angelo BRUCCULERI, La Democrazia cit., p. 102.

(69) Angelo BRUCCULERI, La Democrazia, cit., p. 101. 
e benefício que lhe presta" (70). Alla stessa maniera, se l'individuo, spinto dall'egoismo, viene meno al suo dovere "de respeitar o bem comum, viola a lei da justiça social que protege tanto o Estado quanto os direitos individuais, e portanto renuncia ao direito de fruição do serviço que o Estado presta ao indivíduo" (71).

Concludendo: la violazione dei diritti della persona umana, provenga essa dallo Stato o dall'individuo, è sempre fonte di gravi perturbazioni sociali e di ingiustizie fatali; al contrario, il benessere dello Stato e dell'individuo è garantito nel miglior modo dall'osservanza dei diritti della persona umana.

(70) Guido GONELLA, Bases de uma Ordem Social, cit., p. 180.

(71) Guido GONELLA, Bases de uma Ordem Social, cit., p. 180. 